\title{
A new specific gene for wasp cellular immune resistance in Drosophila
}

\author{
V. BENASSI, F. FREY \& Y. CARTON* \\ Laboratoire Populations, Génétique et Evolution, CNRS 91198 Gif-sur-Yvette Cedex, France
}

\begin{abstract}
Larvae of Drosophila melanogaster produce a haemocytic reaction against eggs of the parasitoid, Asobara tabida, which leads to the formation of a multicellular capsule surrounding the foreign object. The same phenomenon was observed with the parasitoid, Leptopilina boulardi. Concerning the resistance of $D$. melanogaster to $L$. boulardi, a single major segregating locus with the resistant allele dominant to the susceptible one was found. The host strain susceptible to this parasitoid species was found to be highly immune reactive against the eggs of $A$. tabida . The inheritance of the capacity to encapsulate $A$. tabida was analysed by comparing reciprocal crosses made using inbred resistant and susceptible parental strains. We conclude that differences in the encapsulation capacity are inherited autosomally, with the reactive phenotype showing complete dominance over the non-reactive one. These data suggest the existence of two independent gene systems, each being concerned with the recognition of one species only.
\end{abstract}

Keywords: Drosophila, encapsulation, genetic control, immunity, parasitoid.

\section{Introduction}

Little progress has been made during the last decade towards determining the genetic basis of insect cellular resistance to parasite infection, especially that against protozoan or metazoan parasites. Most of the information has been obtained from mosquitoes and fruitflies. Against parasites and other non-self components that are too large to be phagocytosed by individual cells, insects produce a blood cell or haemocytic reaction that leads to the formation of a multicellular capsule surrounding the foreign object. In Aedes aegypti, a broad resistance to multiple disease agents (filaria, Plasmodium, yellow fever virus) appears to be conditional on a single locus or a tightly linked cluster of genes (Severson et al., 1995). Refractoriness to Plasmodium in Anopheles gambiae has been shown to result from two distinct genetic systems (Vernick et al., 1995). The first system, which results in a melanotic encapsulation of ookinetes, is determined by two genetic loci (Collins et al., 1986). The second one, the mechanism of which is a lysis of ookinetes within midgut cells, is determined by a major locus. Whatever the physiological mechanism of resistance or its genetic determinism, no specificity of the immune reaction has

\footnotetext{
*Correspondence. E-mail: carton@hermes.chrs-gif.fr
}

been detected in mosquito immune response, the same 'gene' acting against multiple disease agents.

Regarding resistance of Drosophila melanogaster to the parasitic wasp Leptopilina boulardi (Hymenoptera, Cynipoidea), data obtained from crosses between two host strains showing opposite responses suggested a single major segregating locus, with the resistant allele dominant to the susceptible one (Carton et al., 1992). In contrast to observations in the mosquito, this resistant gene has a high specificity, allowing the recognition and destruction of Leptopilina eggs only. We now have a better understanding of this complex cellular immune reaction developed by Drosophila larvae against the eggs of parasitic wasps (Carton \& Nappi, 1997). The strain susceptible to $L$. boulardi was, however, highly immune reactive against the eggs of Asobara tabida, a wasp species known to infest $D$. melanogaster and D. subobscura in Europe and North America (Kraaijeveld \& van Alphen, 1994). The cellular and biochemical manifestations of the immune response elicited by this $L$. boulardi susceptible strain against A. tabida were identical to those observed in the resistant strain against Leptopilina, resulting in encapsulation of the parasitoid eggs (Nappi et al., 1991, 1992), but were completely absent when the susceptible strain was infested with $L$. boulardi. Thus, the strain susceptible to $L$. boulardi is not 
immune incompetent or otherwise physiologically deficient but is only unable to recognize the eggs of L. boulardi (Carton \& Nappi, 1997).

These data suggest that the hypothesis of two independent gene systems, each determining recognition of a particular wasp species, could be correct. This hypothesis was tested using strains of $D$. melanogaster differing in their ability to produce an immune reaction against an avirulent strain of $A$. tabida. The results obtained from crosses between these two strains also support a single-gene Mendelian model for the resistance of $D$. melanogaster to the braconid $A$. tabida.

\section{Materials and methods}

\section{Origin of strains}

To develop our investigations, two inbred strains of D. melanogaster with opposite immune capacities with respect to the parasitoid $A$. tabida were used. In a previous paper (Vass et al., 1993), it was shown that the strain reactive to $L$. boulardi (Gif stock no. 940 ) is also highly immune reactive against $A$. tabida, rate of encapsulation with $c .95$ per cent; this strain originates from Brazzaville (Africa) and was labelled the $\mathrm{R}$ strain. In choosing the susceptible strain, different laboratory strains were tested and it was discovered that the Canton S strain (Gif stock no. 249) developed no immune reaction against $A$. tabida. This host strain was retained as the susceptible strain and named the $\mathrm{S}$ strain (no encapsulation).

The strain of $A$. tabida, an isofemale line (Gif stock no. $490=$ Leiden stock no. WOV) is derived from a northern European population (Kraaijeveld \& van Alphen, 1994). This strain cannot suppress the immune cellular reaction of $D$. melanogaster.

The flies (D. melanogaster and D. subobscura) were raised on standard cornmeal and dead yeast medium at $25^{\circ} \mathrm{C}$ and $20^{\circ} \mathrm{C}$, respectively. Strain 490 of A. tabida was reared at $20^{\circ} \mathrm{C}$ in population cages. A wild strain of $D$. subobscura (Morocco origin) was used to rear this strain of $A$. tabida, as this host species develops no immune reaction against this wasp species. Adult parasitoids were stored at $14^{\circ} \mathrm{C}$ with honey as food until used for experiments.

\section{Crossing procedure}

Two generations of reciprocal crosses (Table 1) between the resistant (R) and the susceptible (S) strains were performed to yield 10 lines of progeny: two parental strains, two $\mathrm{F}_{1}$ hybrids, four separate backcrosses to both the $\mathrm{S}$ and $\mathrm{R}$ strains and $\mathrm{F}_{2}$ hybrids. Each line was tested for encapsulation capacity at the larval stage, as described in the procedure below.

Table 1 Encapsulation rate of the third instar larvae against Asobara tabida eggs of crosses between resistant (R) and susceptible (S) Drosophila melanogaster strains

\begin{tabular}{|c|c|c|c|c|}
\hline Crosses & Mother $\times$ father & $\begin{array}{l}\text { Number of } \\
\text { replicates }\end{array}$ & $\begin{array}{l}\text { Number of } \\
\text { larvae tested }\end{array}$ & $\begin{array}{l}\text { Encapsulation } \\
\text { rate }(\text { mean } \pm \text { SE) } \\
(\%)\end{array}$ \\
\hline \multicolumn{5}{|c|}{ Parental strains } \\
\hline 1 & $\mathrm{~S} \times \mathrm{S}$ & 7 & 177 & $0.91 \pm 0.53$ \\
\hline 2 & $\mathrm{R} \times \mathrm{R}$ & 7 & 117 & $95.61 \pm 2.63$ \\
\hline \multicolumn{5}{|c|}{ Reciprocal hybrids } \\
\hline 3 & $\mathrm{~S} \times \mathrm{R}$ & 11 & 340 & $87.95 \pm 3.50$ \\
\hline 4 & $\mathrm{R} \times \mathrm{S}$ & 5 & 151 & $92.96 \pm 1.22$ \\
\hline \multicolumn{5}{|c|}{ Reciprocal backcrosses } \\
\hline 5 & $\mathrm{~S} \times(\mathrm{S} \times \mathrm{R})$ & 3 & 120 & $38.83 \pm 3.68$ \\
\hline 6 & $\mathrm{R} \times(\mathrm{S} \times \mathrm{R})$ & 3 & 86 & $94.00 \pm 0.26$ \\
\hline 7 & $(\mathrm{~S} \times \mathrm{R}) \times \mathrm{S}$ & 3 & 124 & $61.70 \pm 5.21$ \\
\hline 8 & $(\mathrm{~S} \times \mathrm{R}) \times \mathrm{R}$ & 4 & 90 & $83.77 \pm 3.99$ \\
\hline \multicolumn{5}{|c|}{ Reciprocal $F_{2}$ hybrids } \\
\hline 9 & $(\mathrm{~S} \times \mathrm{R}) \times(\mathrm{S} \times \mathrm{R})$ & 4 & 163 & $66.48 \pm 4.85$ \\
\hline 10 & $(\mathrm{R} \times \mathrm{S}) \times(\mathrm{R} \times \mathrm{S})$ & 3 & 60 & $85.67 \pm 7.19$ \\
\hline
\end{tabular}




\section{Bioassay procedure}

For every bioassay, five females (between 1 and 2 weeks old) of $A$. tabida were deposited for $8 \mathrm{~h}$ in a plexiglass box containing a batch of 50 host larvae from late second instar or early third instar larvae. The encapsulation ability is less stable and reproducible in late instar larvae $(>24 \mathrm{~h}$ after the third moult). Developmental temperature before infestation was $25^{\circ} \mathrm{C}$. Infestation and rearing of infested larvae were conducted at a temperature of $20^{\circ} \mathrm{C}$. Dissection of infested larvae and determination of the status of the parasitoid egg (encapsulated or not) were carried out 3 days after infestation on late third instar larvae.

The encapsulation rate (percentage) was calculated as the ratio of the number of encapsulated eggs to the total eggs recovered. For each cross, three to 11 replicates (each with 50 larvae submitted to infection) were carried out. Superparasitized larvae were included in the counts, as encapsulation rate does not differ if calculated with monoparasitized or superparasitized larvae.

\section{Statistical analysis}

Analysis of variance (ANOVA) using the general linear model procedure with binomial error and a logit link function (GLIM method; Crawley, 1993) was performed to determine the mode of inheritance of the cellular immune capacity of larvae of $D$. melanogaster against the eggs of $A$. tabida. Comparisons were made according to the methods proposed by Wahlsten (1979) and De Belle \& Sokolowski (1987). This method was used in a previous work on the heritability of resistance to another wasp species (Carton et al., 1992). The following comparisons are referenced in Table 1 and the results are given in Table 2 (the crosses contrasted are in brackets): $\mathrm{S}$ vs. $\mathrm{R}$ parental strains (1 vs. 2 ) to test the differences between the two parental strains; $S+R$ vs. $F_{1}$ to investigate complete genetic dominance or additive effect $(1+2$ vs. $3+4) ; F_{1}$ s for deviation from an autosomal mode of inheritance (3 vs. 4), i.e. non-autosomal inheritance (sex chromosomes, permanent cytoplasmic factors, transient maternal factors).

Test to evaluate a single gene model vs. an additive effect model

We wanted to determine whether the experimental results indicated complete autosomal dominance or a strictly additive pattern of inheritance. The detailed procedure for these comparisons was as given previously (De Belle \& Sokolowski, 1987; Carton et al., 1992). In the case of total dominance, the following order of susceptibilities would be found (Bs: backcross to $\mathrm{S}$; $\mathrm{Br}$ : backcross to $\mathrm{R}$ ):

$\mathrm{S}<\mathrm{Bs}<\mathrm{F}_{2}<\left(\mathrm{F}_{1}=\mathrm{Br}=\mathrm{R}\right)$.

A strictly additive pattern, assuming the effects of resistant and susceptible alleles to be equal but opposite, gives the following order:

$\mathrm{S}<\mathrm{Bs}<\left(\mathrm{F}_{2}=\mathrm{F}_{1}\right)<\mathrm{Br}<\mathrm{R}$.

\section{Test with a single gene model}

As previously described (Carton et al., 1992), the larvae resulting from different crosses were classified as resistant or susceptible, according to their cellular response to the parasitoid egg (immune reactive or

Table 2 Contrast ANOva for encapsulation ability from crosses between resistant (R) and susceptible (S) strains of Drosophila melanogaster (GLIM method for proportion data with a binomial error and a logit link function)

\begin{tabular}{lrrrr}
\hline Source & d.f. & \multicolumn{1}{c}{ MS } & \multicolumn{1}{c}{$F$} & $P$ \\
\hline Model (between crosses) & 9 & 43.55 & 41.48 & 0.0000 \\
Contrasts & & & & \\
$\quad$ 1. S vs. R parental strains (1 vs. 2) & 1 & 191.30 & 182.19 & 0.0000 \\
2. Dominance (1+2 vs. 3+4) & 1 & 150.50 & 143.33 & 0.0000 \\
3. Deviation from an autosomal & 1 & 1.92 & 1.83 & 0.8159 \\
$\quad$ mode of inheritance, i.e. & & & & \\
$\quad$ non-autosomal inheritance (3 vs. 4) & 40 & 1.05 & & \\
$\quad$ Error (within crosses) & 40 &
\end{tabular}


Table 3 Mean encapsulation rate for each reciprocal cross studied and the $t$-test comparison to test the fit to the relationship expected in a one-gene model with complete dominance and non-autosomal inheritance

\begin{tabular}{|c|c|c|c|c|c|c|c|c|c|c|c|}
\hline Cross & $\mathrm{P}_{1}$ & & $\mathrm{BcS}$ & & $\mathrm{F}_{2}$ & & $\mathrm{Bc} R$ & & $\mathrm{~F}_{1}$ & & $\mathrm{P}_{2}$ \\
\hline Expected mean (\%) & 0 & & 50 & & 75 & & 100 & & 100 & & 100 \\
\hline Observed mean (\%) & 0.91 & & 50.28 & & 74.70 & & 88.15 & & 90.93 & & 95.6 \\
\hline$t$-test comparison & & 9.12 & & 3.07 & & 2.18 & & 0.32 & & 1.4 & \\
\hline$P$ & & $0.000^{* *}$ & & $0.011^{*}$ & & $0.049^{*}$ & & $0.751 \mathrm{NS}$ & & $0.156 \mathrm{NS}$ & \\
\hline d.f. & & 11 & & 11 & & 12 & & 21 & & 21 & \\
\hline Cross hierarchy & $\mathrm{P}_{1}$ & $<$ & $\mathrm{Bc} \mathrm{S}$ & $<$ & $\mathrm{F}_{2}$ & $<$ & $\mathrm{Bc} \mathrm{R}$ & $=$ & $\mathrm{F}_{1}$ & $=$ & $\mathrm{P}_{2}$ \\
\hline
\end{tabular}

**Highly significant $(P<0.01)$; * significant $(P<0.05)$; NS, not significant.

non-immune reactive larvae). A reactive larva is one that has encapsulated at least one egg.

Chi-squared analysis of resistant/susceptible observed and expected ratios permitted us to decide whether the data supported a single-gene model with complete dominance.

\section{Results}

\section{Level of encapsulation in the various crosses}

Ten crosses were tested: two parental strains, two $F_{1}$ hybrids, four backcrosses to the $\mathrm{R}$ strain or $\mathrm{S}$ strain and two $\mathrm{F}_{2}$ hybrid strains. The mean encapsulation rates (percentage) \pm standard error (SE) for each cross are presented in Table 1. As previously observed, the larvae of the resistant strain encapsulate most eggs deposited (95.6 per cent), whereas larvae of the S strain were totally unreactive, as they encapsulated less than 1 per cent of parasitic eggs deposited.

\section{Contribution made by autosomes, sex} chromosomes and maternal factors

Contrast analysis of variance allowed us to detect the contribution of chromosomes or other cytoplasmic factors (Table 2). First, as supposed, there is a highly significant difference between resistant and susceptible strains $\left(F_{1,40}=182.2, P<0.000\right)$. Dominance of the resistant character(s) is clearly confirmed by comparison of parental strains with the $\mathrm{F}_{1}$ hybrids $\left(F_{1,40}=143.3, P<0.000\right)$. Reciprocal $\mathrm{F}_{1}$ hybrids do not differ from each other, which indicates that non-autosomal inheritance is not involved $\left(F_{1,40}=1.83\right)$.

\section{The type of inheritance}

In these conditions, the similarity of the encapsulation rate for the resistant parent cross, $\mathrm{Br}$ and $\mathrm{F}_{1}$ implies a typical completely dominant effect verifying the following relationships (Table 3):

$\mathrm{S}<\mathrm{Bs}<\mathrm{F}_{2}<\left(\mathrm{F}_{1}=\mathrm{Br}=\mathrm{R}\right)$.

\section{Testing the fit of a single gene model}

Individuals in the different crosses were distributed into two classes according to their immune capacity (reactive or non-reactive): the resistant class and the susceptible class (Table 4). Non-parametric $2 \times 2$ tables with Yates-corrected chi-squared analysis of resistant/susceptible ratios demonstrate that the observed ratios do not differ significantly from the expected Mendelian ratios in all the crosses observed except in the $\mathrm{S} \times \mathrm{R}$ and $\mathrm{Bc}$ to $\mathrm{R}$ crosses (see below). These data fit a single-gene, complete dominance model of inheritance.

\section{Discussion}

The possession of two inbred lines with opposite responses to $A$. tabida has enabled us to develop a set of crosses, which give new insight into the genetics of resistance of Drosophila larvae to this parasitoid. It may be concluded that differences in the encapsulation capacity of $D$. melanogaster are inherited autosomally, with the resistant phenotype showing complete dominance over the susceptible one. The results of all the crosses suggest a single major segregating locus with two alleles and complete dominance of the resistant allele. It is proposed that the locus encoding for resistance of $D$. melanogaster against $A$. tabida be termed Rat (for Resistance to $A$. tabida) and the resistant and susceptible alleles $\mathrm{Rat}^{+}$and $\mathrm{Rat}^{-}$, respectively. However, it is important to point out that a discrepancy between observed and expected data (Table 4) showing a weak cytoplasmic effect is noticed in the ratio analysis but not in the contrast analysis. Two types of cytoplasmic components are distinguished 
Table 4 Chi-squared analysis of resistant (R) to susceptible (S) larval ratios of Drosophila melanogaster submitted to infection by Asobara tabida

\begin{tabular}{lcccccc}
\hline & $n$ & $\mathrm{R}: \mathrm{S}$ & Expected & Observed & $\chi^{2}$ & $P$ \\
\hline $\begin{array}{l}\text { Parental strains } \\
\quad\end{array}$ & & & & & & \\
$\quad \mathrm{S} \times \mathrm{S}$ & 177 & $0: 1$ & $0: 177$ & $3: 174$ & 1.34 & $0.24 \mathrm{NS}$ \\
$\quad \mathrm{R} \times \mathrm{R}$ & 117 & $1: 0$ & $117: 0$ & $112: 5$ & 3.27 & $0.07 \mathrm{NS}$ \\
$\quad \begin{array}{l}\text { Reciprocal crosses } \\
\quad\end{array}$ & & & & & & \\
$\quad \mathrm{F}_{1} \mathrm{R} \times \mathrm{S}$ & 151 & $1: 0$ & $147: 4$ & $139: 12$ & 3.23 & $0.07 \mathrm{NS}$ \\
$\quad \mathrm{S} \times \mathrm{R}$ & 340 & $1: 0$ & $331: 9$ & $296: 44$ & 23.66 & $0.00^{* *}$ \\
$\quad \mathrm{Bc}$ to $\mathrm{F}$ & & & & & & \\
$\quad \mathrm{F}_{1} \times \mathrm{S}$ & 244 & $1: 1$ & $122: 122$ & $131: 113$ & 0.53 & $0.46 \mathrm{NS}$ \\
$\quad \mathrm{Bc}$ to $\mathrm{R}$ & & & & & & \\
$\quad \mathrm{F}_{1} \times \mathrm{R}$ & 176 & $1: 0$ & $171: 5$ & $161: 15$ & 4.29 & $0.04^{*}$ \\
$\quad \mathrm{~F}_{2}$ & & & & & & \\
$\quad \mathrm{~F}_{1} \times \mathrm{F}_{1}$ & 223 & $3: 1$ & $167: 56$ & $156: 67$ & 1.12 & $0.28 \mathrm{NS}$ \\
\hline
\end{tabular}

Expected ratios are derived by assuming a 5.3 per cent probability of misclassification (obtained from observed ratios in parental $\mathrm{P}_{1}$ and $\mathrm{P}_{2}$ crosses).

**Highly significant $(P<0.01)$; * significant $(P<0.05)$; NS, not significant.

by Wahlsten (1979): permanent and transient cytoplasmic factors. Further investigations will be necessary to confirm this cytoplasmic effect and a possible relationship with the developmental stage of the host larvae. Influences of such factors could also explain the low divergence present in backcrosses to the R strain (Table 4).

It must be pointed out that it is by the acquisition of avirulent strains of parasitic wasps, a situation rarely encountered in the field, that analysis of the Drosophila immune response can be carried out and especially its genetic determination (Carton \& Nappi, 1997). The investigation presented here focused only on the determination of variation in resistance of $D$. melanogaster, using two lines selected for their opposite response to a uniquely unprotected isofemale line of $A$. tabida. In any case, this work could resolve the genetic interplay and local genetic adaptation observed between the sympatric populations of $D$. melanogaster and $A$. tabida.

In a previous paper (Carton et al., 1992), we detected that a single major segregating locus with two alleles and complete dominance of the resistant allele, determines the resistance of the same host species, i.e. D. melanogaster, to another wasp species, L. boulardi.

The question arises, then, as to whether the same genetic system works against the two wasp species, even if these species belong to different families of Hymenoptera: Braconiidae and Cynipoidae. In fact, we have shown (Vass et al., 1993) that the strain of $D$. melanogaster that is susceptible to $L$. boulardi is highly immune reactive against $A$. tabida. It was also possible to obtain two other $D$. melanogaster strains, one equally resistant against the two parasitoids and the other one totally susceptible. This could suggest that the responses of the same host strain to the two parasitoids are totally non-correlated and that the two genetic factors are independent. Additional evidence to support the proposal of two independent genes, each conferring a specific immune resistance, is provided by the immune responses of about 30 natural European populations of D. melanogaster, which show no correlation between a strain's capacity to encapsulate $L$. boulardi or A. tabida (Kraaijeveld \& van Alphen, 1995).

It is also necessary to identify the particular step in the complex pathway of the immune cellular reaction against a parasitoid with which these resistance genes interfere. It is now clear that differences in immune reactivity between the resistant and susceptible lines could not be attributed to differential abilities of melanizing enzymes and/or encapsulation capacity (Carton \& Nappi, 1997). The strain susceptible to one parasitoid species appears to be totally immune reactive to a second species. Apparent high specificity of the resistance gene product suggests that it could play a key role in response induction. Whatever determines the target specificity of the resistance gene action is certainly acting at an early stage in the reaction, i.e. during the recognition 
process. It is known that invertebrate immunity, especially in lower metazoan phyla, exhibits rapid cellular recognition without prior contact (Humphreys \& Reinherz, 1994). Early phases of host defence certainly involve receptors and ligands. It has been proposed (Janeway, 1992) that primitive effector cells bear pattern recognition receptors. Unfortunately, the nature of the putative receptors that recognize some generic cell surface ligands and their locations are entirely speculative (Carton \& Nappi, 1997). Antibacterial response can be induced after lipopolysaccharide (LPS) injection. Recently, $\mathrm{Xu}$ et al. (1995) identified an LPS-binding protein in haemocytes, which might relate to the specific membrane receptor for LPS. This binding site is specific for some responsive cells and is located on the cell surface. Another candidate has also been suggested as the recognition structure, the Drosophila scavenger receptors (Pearson et al., 1995). We can suggest as a first hypothesis for future investigations that these genes for resistance are also good candidates as coding for recognition structures in Drosophila.

\section{Acknow ledgements}

We would like to thank A. R. Kraaijeveld and J. J. M. van Alphen (Leiden University) for providing the strain of $A$. tabida, and P. Chabora and A. Nappi for careful reading of the manuscript. This work was supported by an ACC programme (MESR, France) and by an EC programme (CEE-AIR3-CT9-1433).

\section{References}

CARTON, Y. AND NAPPI, A. J. 1997. Drosophila cellular immunity against parasitoids. Parasitol. Today, 13, 218-226.

CARTON, Y., FREY, F. AND NAPPI, A. 1992. Inheritance of cellular immune resistance in Drosophila melanogaster. Heredity, 69, 393-399.

COLLINS, F. H., SAKAI, R. K., VERNICK, K. D., PASKEWITZ, S., SEELEY, D. C., MILLER, L. H. ET AL. 1986. Genetic selection of a Plasmodium-refractory strain of the malaria vector Anopheles gambiae. Science, 234, 607-610.

CRAWLEY, M. J. 1993. GLIM for Ecologists. In: Lawton, J. H. and Likens, G. E. (eds) Methods in Ecology, Series 15, pp. 1-379. Blackwell Scientific Publications, Oxford. DE BELLE, S. AND SOKOLOWSKI, M. B. 1987. Heredity of rover/sitter: alternative foraging strategies of Drosophila melanogaster larvae. Heredity, 59, 73-83.
HUMPHREYS, T. AND REINHERZ, E. L. 1994. Invertebrate immune recognition, natural immunity and the evolution of positive selection. Immunol. Today, 15, 316-320.

JANEWAY, C. A. 1992. The immune system evolved to discriminate infectious nonself from noninfectious self. Immunol. Today, 13, 11-16.

KRAAIJEVELD, A. R. AND VAN ALPHEN, J. J. M. 1994. Geographical variation in resistance of the parasitoid $A$. tabida against encapsulation by Drosophila melanogaster larvae: the mechanism explored. Physiol. Entomol., 19, 9-14.

KRAAIJEVELD, A. R. AND VAN ALPHEN, J. J. M. 1995. Geographical variation in encapsulation ability of Drosophila melanogaster larvae and evidence for parasitoid-specific components. Evol. Ecol., 9, 10-17.

NAPPI, A. J., CARTON, Y. AND FREY, F. 1991. Parasiteinduced enhancement of hemolymph tyrosinase activity in a selected immune reactive strain of Drosophila melanogaster. Arch. Insect Biochem. Physiol., 18, 159-168.

NAPPI, A. J., VASS, E., CARTON, Y. AND FREY, F. 1992. Identification of 3, 4-dihydroxyphenylalanine, 5, 6-dihroxyindole and $\mathrm{N}$-acetylarterenone during eumelanin formation in immune reactive larvae of Drosophila melanogaster. Arch. Insect Biochem. Physiol., 10, 181-191.

PEARSON, A., LUX, A. AND KRIEGER, M. 1995. Expression cloning of dSR-CI, a class $\mathrm{C}$ macrophage-specific scavenger receptor from Drosophila melanogaster. Proc. Natl. Acad. Sci. U.S.A., 92, 4056-4060.

SEVERSON, D. H., THATHY, V. V., MORI, A. ZHANG, Y. AND CHRISTENSEN, B. M. 1995. Restriction fragment length polymorphism mapping of quantitative trait loci for malaria parasite susceptibility in the mosquito Aedes aegypti. Genetics, 139, 1711-1717.

VASS, E., NAPPI, A. J. AND CARTON, Y. 1993. Comparative study of immune competence and host susceptibility in Drosophila melanogaster parasitized by Leptopilina boulardi and A. tabida. J. Parasitol., 79, 106-112.

VERNICK, K. D., FUJOKA, H., SEELEY, D. C., TANDLER, B., AIKAWA, M. AND MILlER, L. H. 1995. Plasmodium gallicanum: a refractory mechanism of ookinete killing in the mosquito, Anopheles gambiae. Exp. Parasitol., 80, 563-595.

WAHLSTEN, D. 1979. A critique of the concepts of heritability and heredity in behavioral genetics. In: Royce, J. R. and Mos, L. (eds) Theoretical Advances in Behavioral Genetics, pp. 426-481. Sithoff and Nordhoff, Germantown, MD.

XU, J., NISHIJIMA, M., KONO, Y., TANIAL, K., KATO, Y., KADONO-OKUDA, K. ET AL. 1995. Identification of a hemocyte membrane protein of the silkworm, Bombyx mori, which specifically binds to bacterial lipopolysaccharide. Insect Biochem. Mol. Biol., 25, 921-928. 